\title{
Multicolor on-line degree Ramsey numbers of trees
}

\author{
William B. KinnersleY* AND Douglas B. West ${ }^{\dagger}$
}

In the on-line Ramsey game on a family $\mathcal{H}$ of graphs, "Builder" presents edges of a graph one-by-one, and "Painter" colors each edge as it is presented; we require that Builder keep the presented graph in $\mathcal{H}$. Builder wins the game $(G, \mathcal{H})$ if Builder can ensure that a monochromatic $G$ arises. The $s$-color on-line degree Ramsey number of $G$, denoted $\stackrel{\circ}{R}_{\Delta}(G ; s)$, is the least $k$ such that Builder wins $(G, \mathcal{H})$ when $\mathcal{H}$ is the family of graphs having maximum degree at most $k$ and Painter has $s$ colors available. More generally, $R_{\Delta}\left(G_{1}, \ldots, G_{s}\right)$ is the minimum $k$ such that Builder can force a copy of $G_{i}$ in color $i$ for some $i$ when restricted to graphs with maximum degree at most $k$.

In this paper, we prove that $\stackrel{\circ}{R}_{\Delta}(T ; s) \leq s(\Delta(T)-1)+1$ for every tree $T$; this is sharp, with equality whenever $T$ has adjacent vertices of maximum degree. We also give lower and upper bounds on $\stackrel{\circ}{R}_{\Delta}\left(G_{1}, \ldots, G_{s}\right)$ when each $G_{i}$ is a double-star. When each $G_{i}$ is a star, we determine $\stackrel{\circ}{R}_{\Delta}\left(G_{1}, \ldots, G_{s}\right)$ exactly.

\section{Introduction}

When every 2-edge-coloring of a host graph $H$ contains a monochromatic copy of a target graph $G$, we write $H \rightarrow G$. More generally, when every $s$-edge-coloring of $H$ contains a monochromatic $G$, we write $H \stackrel{s}{\rightarrow} G$. The central problem of graph Ramsey theory is to find the least $n$ such that $K_{n} \rightarrow G$, which can be restated as $\min \{|V(H)|: H \rightarrow G\}$. The value is called the Ramsey number of $G$, denoted $R(G)$ (or $R(G ; s)$ in the $s$-color setting).

This phrasing of the Ramsey number generalizes: given a graph parameter $\rho$, let $R_{\rho}(G)=\min \{\rho(H): H \rightarrow G\}$. When $\rho$ is the maximum degree, the Ramsey parameter is the degree Ramsey number, $R_{\Delta}(G)$. Burr, Erdös, and

*Research supported in part by National Science Foundation grant DMS 0838434 "EMSW21-MCTP: Research Experience for Graduate Students".

${ }^{\dagger}$ Research supported in part by the National Security Agency under Award No. H98230-10-1-0363. 
Lovász [2] introduced this notion and determined $R_{\Delta}\left(K_{1, k}\right)$ and $R_{\Delta}\left(K_{n}\right)$; further results appear in $[7,9]$.

An on-line variant of the degree Ramsey number can be phrased as a game played by two players, "Builder" and "Painter", on an infinite set of vertices. In each round, Builder introduces an edge and Painter colors it from a fixed set of $s$ colors. Builder aims to force a monochromatic copy of a target graph $G$. By Ramsey's Theorem, Builder can win by presenting a large complete graph. Thus we restrict Builder by requiring that the presented graph remains in a family $\mathcal{H}$; the game is then played on $\mathcal{H}$. If Builder can still force a monochromatic $G$, then we say Builder wins. More generally, Builder wins $\left(G_{1}, \ldots, G_{s} ; \mathcal{H}\right)$ if Builder can force a copy of $G_{i}$ in color $i$ for some $i$ when playing on $\mathcal{H}$ with Painter having $s$ colors available. Introduced by Beck [1], this model was studied by Grytczuk, Hałuszczak, and Kierstead [5] for several natural choices of $\mathcal{H}$ in the case where $s=2$ and $G_{1}=G_{2}$. Later results appear in $[4,6,8,10]$.

We focus on the case where $\mathcal{H}$ is $\mathcal{S}_{k}$, the set of graphs with maximum degree at most $k$. We define $\stackrel{\circ}{R}_{\Delta}\left(G_{1}, \ldots, G_{s}\right)$ to be the least $k$ such that Builder wins $\left(G_{1}, \ldots, G_{s} ; \mathcal{S}_{k}\right)$. When $G_{1}=\cdots=G_{s}=G$, we have the diagonal case and abbreviate the notation to $\stackrel{\circ}{R}_{\Delta}(G ; s)$, called the $s$-color on-line degree Ramsey number of $G$. The parameter is well-defined, since it is bounded by the ordinary $s$-color Ramsey number minus 1 .

For $s=2$, Butterfield et al. [3] determined the exact 2-color on-line degree Ramsey numbers for paths, stars, and double-stars (trees with diameter 3 ), and they proved that $\stackrel{\circ}{R}_{\Delta}(T ; 2) \leq 2 \Delta(T)-1$ for every tree $T$. In this paper, we extend several of those results to the $s$-color, non-diagonal setting. Proposition 2.3 states that $\stackrel{\circ}{R}_{\Delta}\left(P_{n_{1}}, \ldots, P_{n_{s}}\right)=s+1$ when each $n_{i}$ is at least 4 , where $P_{n}$ denotes the path with $n$ vertices. This result uses a recursive lower bound for $\stackrel{\circ}{R}_{\Delta}\left(G_{1}, \ldots, G_{s}\right)$ in terms of $\stackrel{\circ}{R}_{\Delta}\left(G_{1}, \ldots, G_{s-1}\right)$. Theorem 2.5 gives somewhat technical lower and upper bounds for $\stackrel{\circ}{R}_{\Delta}\left(G_{1}, \ldots, G_{s}\right)$ when each $G_{s}$ is a double-star; these bounds coincide when the central vertices of each $G_{i}$ have identical degrees (Corollary 2.7). A refined argument determines the exact value when each $G_{i}$ is a star (Theorem 2.8). Finally, Theorem 2.10 states that $\stackrel{\circ}{R}_{\Delta}\left(T_{1}, \ldots, T_{s}\right) \leq \sum_{i=1}^{s}\left(\Delta\left(T_{i}\right)-1\right)+1$ when each $T_{i}$ is a tree; this bound holds with equality when each $T_{i}$ has adjacent vertices of maximum degree.

\section{Results}

In the course of a particular game, we often focus attention on special subgraphs of the presented graph, usually monochromatic. In such situations, 
we must distinguish between the degree of a vertex within the subgraph and its degree within the full presented graph. We use "degree" to mean "degree within the given subgraph" and "global degree" to mean "degree within the full presented graph".

In giving strategies for Builder to prove upper bounds, we may assume that Painter behaves "consistently". A consistent Painter chooses a color for edge $u v$ based solely on the edge-colored components presently containing $u$ and $v$. It was shown in [3] that for any graph $G$ and any monotone additive graph family $\mathcal{H}$, Builder wins $(G ; \mathcal{H})$ if and only if Builder wins against every consistent Painter. Thus consistent Painters are no weaker than general Painters, but this formal restriction on the Painter simplifies what needs to be said for a Builder strategy. If Builder repeats the same sequence of moves on disjoint sets of vertices, then a consistent Painter produces the same coloring every time. This observation yields the lemma below, which we apply throughout the paper without explicit citation.

Lemma 2.1. If Builder can force an edge-colored graph $G$ against a consistent Painter, then Builder can force arbitrarily many copies of $G$.

We often apply Lemma 2.1 when presenting strategies for Builder. Builder maintains "partial" copies of the target graphs in each color, uses Lemma 2.1 to produce many of these partial copies, and presents edges joining them in such a way that Painter must either augment one of the copies or directly produce a monochromatic copy of some target graph. Eventually, one of the partial copies grows to include the full target graph, and Builder wins.

Our first result is a general lower bound on $\stackrel{\circ}{R}_{\Delta}(G ; s)$. It uses a Painter strategy that generalizes the "greedy $\mathcal{S}_{k}$-Painter" from [3], who colors an edge red when the resulting red subgraph would belong to $\mathcal{S}_{k}$ and blue otherwise.

Proposition 2.2. For graphs $G_{1}, \ldots, G_{s}$,

$\stackrel{\circ}{R}_{\Delta}\left(G_{1}, \ldots, G_{s}\right) \geq\left(\stackrel{\circ}{R}_{\Delta}\left(G_{1}, \ldots, G_{s-1}\right)-1\right)+\max _{u v \in E\left(G_{s}\right)} \min \left\{d_{G_{s}}(u), d_{G_{s}}(v)\right\}$.

Proof. Let $r=\stackrel{\circ}{R}_{\Delta}\left(G_{1}, \ldots, G_{s-1}\right)-1$ and $d=\max _{u v \in E\left(G_{s}\right)} \min \left\{d_{G_{s}}(u)\right.$, $\left.d_{G_{s}}(v)\right\}$; we provide a strategy for Painter to win on $\mathcal{S}_{r+d-1}$. Painter colors edges using blue and $s-1$ shades of red. Painter behaves similarly to a greedy $\mathcal{S}_{r}$-Painter. However, whenever Painter colors an edge red, he chooses the particular shade of red according to some winning strategy for $\left(G_{1}, \ldots, G_{s-1} ; \mathcal{S}_{r}\right)$. In this way Painter avoids producing a copy of any $G_{i}$ in the corresponding shade of red; it suffices to show that also Painter produces no blue $G_{s}$. 
Suppose that Painter has produced a blue copy $H$ of $G_{s}$. Choose an edge $u v$ in $H$ maximizing $\min \left\{d_{H}(u), d_{H}(v)\right\}$. Since Painter colored $u v$ blue, one of its endpoints, say $u$, lies on $r$ red edges in the presented graph. Since $u$ also lies on at least $d_{H}(u)$ blue edges, it has global degree at least $r+d$, a contradiction.

As an application of Proposition 2.2, we determine $\stackrel{\circ}{R}_{\Delta}\left(P_{n_{1}}, \ldots, P_{n_{s}}\right)$; the proof of this result introduces techniques used in the proof of Theorem 2.10.

Proposition 2.3. $\stackrel{\circ}{R}_{\Delta}\left(P_{n_{1}}, \ldots, P_{n_{s}}\right)=s+1$ when $n_{i} \geq 4$ for all $i$ in $\{1, \ldots, s\}$.

Proof. The lower bound follows from Proposition 2.2 and the observations that $\stackrel{\circ}{R}_{\Delta}\left(P_{n} ; 1\right)=2$ and that the maximum over $u v \in E\left(P_{n}\right)$ of $\min \left\{d_{P_{n}}(u)\right.$, $\left.d_{P_{n}}(v)\right\}$ is 2 when $n \geq 4$.

For the upper bound, it suffices to prove that $\stackrel{\circ}{R}_{\Delta}\left(P_{n} ; s\right)=s+1$ when $n \geq 4$, where $n=\max \left\{n_{1}, \ldots, n_{s}\right\}$. We provide a strategy for Builder by induction on $s$; note again that $\stackrel{\circ}{R}_{\Delta}\left(P_{n} ; 1\right)=2$. Suppose that Builder can force $P_{n}$ on $\mathcal{S}_{s}$ when Painter has $s-1$ colors available. Consider an $s$-color game on $\mathcal{S}_{s+1}$. A consistent Painter uses the same color on all isolated edges; without loss of generality, call this color blue. Let the other $s-1$ colors be shades of red. It suffices to show that for any $k$, Builder can force either a $P_{n}$ in some shade of red or a blue $P_{2^{k}}$ in which each endpoint has global degree 1.

We prove this claim by induction on $k$. The case $k=1$ is immediate, since Painter colors isolated edges blue. For the induction step, Builder first forces many blue copies of $P_{2^{k-1}}$ whose endpoints have global degree 1 . Builder next selects one endpoint from each of these blue paths. On these endpoints, Builder plays a winning strategy for the $(s-1)$-color game $\left(P_{n} ; \mathcal{S}_{s}\right)$, provided by the overall induction hypothesis (the global degree remains at most $s+1$ ). If Painter uses only the $s-1$ shades of red, then $P_{n}$ arises in some shade of red. Otherwise, Painter colors some edge blue; this connects two blue paths, yielding a blue $P_{2^{k}}$ whose endpoints still have global degree 1 .

We next consider stars and double-stars.

Definition 2.4. A double-star is a tree with diameter 3. Such a tree has two central vertices; we denote by $S_{a, b}$ the double-star with central vertices of degrees $a$ and $b$. 
Theorem 2.5. If $a_{i} \leq b_{i}$ for all $i$ in $\{1, \ldots, s\}$, then

$$
\begin{aligned}
b_{1}-1+\sum_{i=2}^{s}\left(a_{i}-1\right)+1 & \leq \stackrel{\circ}{R}_{\Delta}\left(S_{a_{1}, b_{1}}, \ldots, S_{a_{s}, b_{s}}\right) \\
& \leq \min _{X \subseteq\{1, \ldots, s\}} f_{X}\left(a_{1}, \ldots, a_{s}, b_{1}, \ldots, b_{s}\right),
\end{aligned}
$$

where

$$
\begin{aligned}
& f_{X}\left(a_{1}, \ldots, a_{s}, b_{1}, \ldots, b_{s}\right) \\
& \quad=1+\max \left\{\sum_{i \in X}\left(b_{i}-1\right)+\sum_{j \notin X}\left(a_{j}-1\right), \sum_{i \in X}\left(a_{i}-1\right)+\sum_{j \notin X}\left(b_{j}-1\right)\right\} .
\end{aligned}
$$

Proof. The lower bound follows by induction on $s$, using Proposition 2.2 and the observation that $\stackrel{\circ}{R}_{\Delta}\left(S_{a, b} ; 1\right)=b$ when $a \leq b$.

To establish the upper bound, we provide a strategy for Builder. Builder first partitions the set of available colors into some sets $X$ and $Y$. Builder aims to make two special vertices $u$ and $v$ the central vertices of a monochromatic double-star. Let the quota of $u$ in color $i$ be $b_{i}-1$ if $i \in X$ and $a_{i}-1$ if $i \in Y$. For $v$, use the reverse values: the quota of $v$ in color $i$ is $a_{i}-1$ if $i \in X$ and $b_{i}-1$ if $i \in Y$. Whenever $u$ or $v$ reaches its quota of incident edges in a color $c$, call that vertex saturated in color $c$. Note that coloring $u v$ with a color in which both $u$ and $v$ are saturated produces the desired monochromatic double-star in that color.

Starting with $u$ and $v$ as isolated vertices, Builder repeats the following process for the remainder of the game. Let $G_{u}$ and $G_{v}$ denote the current components of the presented graph that contain $u$ and $v$, respectively. Builder presents edge $u v$; let $c$ be the color Painter uses on it. If $u$ was not already saturated in $c$, then Builder adds $u v$ and all of $G_{v}$ to $G_{u}$, creates new copies of $v$ and $G_{v}$, and repeats. If $u$ was saturated in $c$ but $v$ was not, then Builder adds $u v$ and all of $G_{u}$ to $G_{v}$, creates new copies of $u$ and $G_{u}$, and repeats. Finally, if both $u$ and $v$ were already saturated in $c$, then $u$ and $v$ are now the central vertices of a monochromatic $S_{a_{c}, b_{c}}$ in color $c$, and Builder has won.

Whenever $G_{u}$ or $G_{v}$ is enlarged, the special vertex receives another incident edge, so always $u$ or $v$ has maximum global degree after $G_{u}$ and $G_{v}$ are "recreated". When Builder is ready to present the edge $u v$, the degree of $u$ is at most $\sum_{i \in X}\left(b_{i}-1\right)+\sum_{j \notin X}\left(a_{j}-1\right)$, and the degree of $v$ is at most $\sum_{i \in X}\left(a_{i}-1\right)+\sum_{j \notin X}\left(b_{j}-1\right)$. Thus the maximum degree used is at most one more than the maximum of these two quantities. Optimizing over the choice of $X$ yields the stated bound. 
In the diagonal case, the minimum over $X$ in the upper bound in Theorem 2.5 occurs whenever $|X|=\lceil s / 2\rceil$. This yields a much simpler formula:

Corollary 2.6. If $a \leq b$, then $\stackrel{\circ}{R}_{\Delta}\left(S_{a, b} ; s\right) \leq\lceil s / 2\rceil(b-1)+\lfloor s / 2\rfloor(a-1)+1$.

When $a_{i}=b_{i}$ for all $i$, the upper and lower bounds in Theorem 2.5 coincide:

Corollary 2.7. For "symmmetric" double stars, $\stackrel{\circ}{R}_{\Delta}\left(S_{b_{1}, b_{1}}, \ldots, S_{b_{s}, b_{s}}\right)=$ $\sum_{i=1}^{s}\left(b_{i}-1\right)+1$. In particular, $\stackrel{\circ}{R}_{\Delta}\left(S_{b, b} ; s\right)=s(b-1)+1$.

When each double-star is in fact a star, the upper bound in Theorem 2.5 is the correct value. The answer is obtained by splitting the sum of the sizes of the stars as equally as possible and using the larger half in such a split.

Theorem 2.8. $\stackrel{\circ}{R}_{\Delta}\left(K_{1, k_{1}}, \ldots, K_{1, k_{s}}\right)=1+\min _{X \subseteq\{1, \ldots, s\}} \max \left\{\sum_{i \in X}\left(k_{i}-1\right)\right.$, $\left.\sum_{i \notin X}\left(k_{i}-1\right)\right\}$. In particular, $\stackrel{\circ}{R}_{\Delta}\left(K_{1, k} ; s\right)=\left\lceil\frac{s}{2}\right\rceil(k-1)+1$.

Proof. The upper bound follows from Theorem 2.5.

For the lower bound, we provide a strategy for Painter to win on $\mathcal{S}_{d-1}$, where $d$ is the claimed bound. Call a vertex saturated in color $i$ when it lies on $k_{i}-1$ edges of color $i$. Painter's strategy is straightforward: when Builder presents an edge, Painter colors it with any color in which neither endpoint is already saturated.

To show that this is always possible, consider the possibility of Builder playing an edge $u v$. If no color is available for use on $u v$, then for each $i$, either $u$ or $v$ is saturated in color $i$. Let $X$ be the set of colors in which $u$ is saturated; $u$ has degree at least $\sum_{i \in X}\left(k_{i}-1\right)$. Likewise, since $v$ is saturated in the remaining colors, $v$ has degree at least $\sum_{i \notin X}\left(k_{i}-1\right)$. Thus $u$ or $v$ already has degree at least $d-1$, and Builder cannot present $u v$.

The lower bound in Theorem 2.8 yields a general lower bound:

Corollary 2.9. $\stackrel{\circ}{R}_{\Delta}\left(G_{1}, \ldots, G_{s}\right) \geq 1+\min _{X \subseteq\{1, \ldots, s\}} \max \left\{\sum_{i \in X}\left(k_{i}-1\right)\right.$, $\left.\sum_{i \notin X}\left(k_{i}-1\right)\right\}$, where $k_{i}=\Delta\left(G_{i}\right)$ for $1 \leq i \leq s$.

Proof. The on-line degree Ramsey number is monotone: if $G_{i}^{\prime} \subseteq G_{i}$ for $1 \leq$ $i \leq s$, then $\stackrel{\circ}{R}_{\Delta}\left(G_{1}, \ldots, G_{s}\right) \geq \stackrel{\circ}{R}_{\Delta}\left(G_{1}^{\prime}, \ldots, G_{s}^{\prime}\right)$. Consequently, $\stackrel{\circ}{R}_{\Delta}\left(G_{1}, \ldots\right.$, $\left.G_{s}\right) \geq \stackrel{\circ}{R}_{\Delta}\left(K_{1, k_{1}}, \ldots, K_{1, k_{s}}\right)$, and Theorem 2.8 applies.

We next turn to general trees. Corollary 2.7 shows that $\stackrel{\circ}{R}_{\Delta}\left(S_{b, b} ; s\right)=$ $s(b-1)+1$; in fact, this is the maximum value of $\stackrel{\circ}{R}_{\Delta}(T ; s)$ over all trees with maximum degree $b$. This was shown in [3] for $s=2$; we prove the general result by a different approach. 
Theorem 2.10. If $T_{1}, \ldots, T_{s}$ are trees, then $\stackrel{\circ}{R}_{\Delta}\left(T_{1}, \ldots, T_{s}\right) \leq \sum_{i=1}^{s}\left(\Delta\left(T_{i}\right)-\right.$ $1)+1$. Moreover, the bound holds with equality whenever all $T_{i}$ have adjacent vertices of maximum degree.

Proof. The sharpness follows from Corollary 2.7 and the monotonicity of $\stackrel{\circ}{R}_{\Delta}$.

For the upper bound, we provide a strategy for Builder. To simplify notation, let $d$ be the claimed bound, let $k_{i}=\Delta\left(T_{i}\right)$, and let $h_{i}=\left|V\left(T_{i}\right)\right|$. If each $k_{i}$ is 1 , then Builder wins by presenting a single edge. We proceed by induction on $\sum_{i} k_{i}$. If any $k_{i}$ is 1 , then $T_{i}$ is a single edge, so color $i$ may be ignored: if Painter ever uses that color, then Builder wins. Thus Builder wins by following a strategy to win $\left(T_{1}, \ldots, T_{i-1}, T_{i+1}, \ldots, T_{s} ; \mathcal{S}_{d}\right)$, the existence of which is guaranteed by the induction hypothesis.

Hence we may assume $k_{i} \geq 2$ for each $i$. Let $T^{k, h}$ denote the rooted tree in which all non-leaves have degree $k$ and all leaves lie at distance $h$ from the root. Since $T_{i} \subseteq T^{k_{i}, h_{i}}$ for each $i$, by monotonicity it suffices to show that $\stackrel{\circ}{R}_{\Delta}\left(T^{k_{1}, h_{1}}, \ldots, T^{k_{s}, h_{s}}\right) \leq \sum_{i}\left(k_{i}-1\right)+1$.

Builder aims to grow a tree containing $T^{k_{i}, h_{i}}$ in color $i$, for some $i$. More generally, let a $(k, h)$-subtree be a rooted tree with the property that all non-leaves within distance $h$ of the root have degree $k$ in the tree and all leaves within distance $h$ of the root have global degree 1. Builder can force a $\left(k_{i}, h_{i}\right)$-subtree in color $i$ for some $i$ by playing a star with up to $d$ edges. The Pigeonhole Principle yields a star with $k_{i}$ edges in color $i$ for some $i$ by the time this is finished, and such a star is a $\left(k_{i}, h_{i}\right)$-subtree.

It now suffices to show that if Builder can force a $\left(k_{i}, h_{i}\right)$-subtree $T$ in color $i$, then he can either win or force a $\left(k_{i}, h_{i}\right)$-subtree $T^{\prime}$ in color $i$ that has more vertices than $T$ within distance $h_{i}$ of the root. This completes the proof because the number of vertices within distance $h_{i}$ of the root of a $\left(k_{i}, h_{i}\right)$-subtree is maximized when the tree contains $T^{k_{i}, h_{i}}$.

Without loss of generality, we may assume that the monochromatic star produced by Painter when Builder starts the process with an isolated star has color 1, which we call red.

Let $v$ be the root of the current red $\left(k_{1}, h_{1}\right)$-subtree, $T$. If $T$ has no leaves with distance less than $h_{1}$ from $v$, then $T$ contains $T^{k_{1}, h_{1}}$, and Builder wins. Otherwise, let $x$ be some such leaf. Builder forces many copies of $T$ (Builder plays against a consistent Painter). We consider two cases.

Case 1: $k_{1} \geq 3$. By the induction hypothesis, Builder has a strategy to win the game $\left(T^{k_{1}-1, h_{1}}, T^{k_{2}, h_{2}}, \ldots, T^{k_{s}, h_{s}} ; \mathcal{S}_{d-1}\right)$; Builder plays this strategy on the copies of $x$ within the copies of $T$. Since each copy of $x$ had global degree 1 when its copy of $T$ was created, the presented graph remains within $\mathcal{S}_{d}$. Builder either wins the original game (if the threshold is reached in 


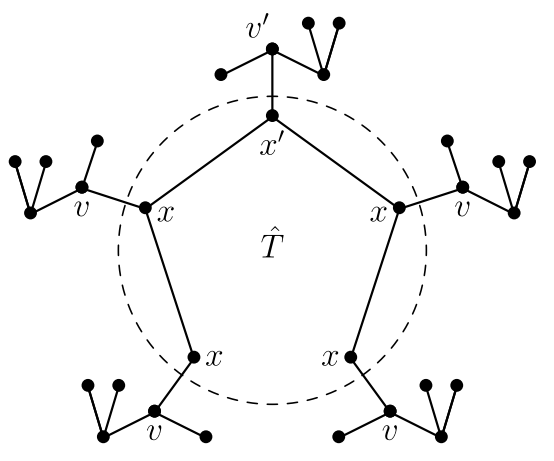

Figure 1: Induction step for Case 1 of Theorem $2.10\left(k_{1}=3\right.$ and $\left.h_{1}=2\right)$.

another color) or forces a red $T^{k_{1}-1, h_{1}}$ (see Figure 1 , where $k_{1}=3$ and $\left.h_{1}=2\right)$.

In the latter case, let $\hat{T}$ be the red copy of $T^{k_{1}-1, h_{1}}$ produced, and let $x^{\prime}$ be its root. Let $v^{\prime}$ be the copy of $v$ within the copy of $T$ containing $x^{\prime}$, and let $T^{\prime}$ be the maximal red tree rooted at $v^{\prime}$ (in Figure 1, all edges drawn belong to $T^{\prime}$ ). All non-leaves of $T^{\prime}$ within distance $h_{1}$ of $v^{\prime}$ lie on $k_{1}$ red edges: those that were leaves in their copies of $T$ lie on $k_{1}-1$ red edges from $\hat{T}$ and one from $T$, while all others were non-leaves in their copies of $T$. Since leaves of $\hat{T}$ lie at distance $h_{1}$ from $x^{\prime}$, their distances from $v^{\prime}$ exceed $h_{1}$, so their degrees in red are unimportant. Every leaf of $T^{\prime}$ within distance $h_{1}$ of $v^{\prime}$ has global degree 1 , because each corresponds to a leaf in its copy of $T$. Note that $T^{\prime}$ has more vertices within distance $h_{1}$ of $v^{\prime}$ than $T$ has within distance $h_{1}$ of its root, since $x^{\prime}$ acquires children in $\hat{T}$. Thus $T^{\prime}$ with root $v^{\prime}$ is the desired $\left(k_{1}, h_{1}\right)$-subtree.

Case 2: $k_{1}=2$. Builder cannot proceed as before, because $T^{1, h_{1}}$ may not be well-defined. Note that $T^{2, h_{1}}$ is a path. Since $k_{1}=2$, in the initial phase Builder can force many red copies of $P_{3}$ whose endpoints have global degree 1 . Builder plays a winning strategy for $\left(P_{2}, T^{k_{2}, h_{2}}, \ldots, T^{k_{s}, h_{s}} ; \mathcal{S}_{d-1}\right)$ on copies of $x$, where $x$ is a leaf of the current red path that Builder can force (with endpoints of global degree 1). If Painter uses no red edges, then Builder wins, by the induction hypothesis. Otherwise, Builder obtains a longer red path in which both endpoints have global degree 1 ; he then chooses $x$ to be one of these endpoints and repeats the process as needed, eventually either winning or obtaining a red $T^{2, h_{1}}$.

In the diagonal case, the bound reduces to a simpler expression:

Corollary 2.11. If $T$ is a tree, then $\stackrel{\circ}{R}_{\Delta}(T ; s) \leq s(\Delta(T)-1)+1$. 
The double-star $S_{b, b}$ shows that Corollary 2.11 is sharp. However, we do not know whether the bound is sharp for any tree not having adjacent vertices of maximum degree.

Comparing $\stackrel{\circ}{R}_{\Delta}$ and $R_{\Delta}$, we remark that $R_{\Delta}(T) \leq 2 s(\Delta(T)-1)$ for any tree $T$ [7], and it was shown in [9] that this bound is asymptotically tight. Thus the maximum value of the on-line degree Ramsey number over the class of trees is about half that of the "off-line" degree Ramsey number. It would be interesting to know in general what affects the ratio between the two parameters.

\section{References}

[1] Beck, J. (1993). Achievement games and the probabilistic method, in: Combinatorics, Paul Erdös is Eighty, vol. 1, Bolyai Soc. Math. Stud. 51-78. MR1249704

[2] Burr, S., Erdős, P. and Lovász, L. (1976). On graphs of Ramsey type. Ars Combinatoria 1 167-190. MR0419285

[3] Butterfield, J., Grauman, T., Kinnersley, W. B., Milans, K. G., Stocker, C., and West, D. B. (2011). On-line Ramsey theory for bounded-degree graphs. Electron. J. Combin. 18 Research Paper 136, 17 pp. (electronic). MR2817786

[4] Conlon, D. (2009/2010). On-line Ramsey numbers. SIAM J. Discrete Math. 23 1954-1963. MR2594965

[5] Grytczuk, J. A., Hałuszczak, M. and Kierstead, H. A. (2004). On-line Ramsey theory. Electron. J. Combin. 11 Research Paper 57, 10 pp. (electronic). MR2097326

[6] Grytczuk, J. A., Kierstead, H. A. and Prałat, P. (2008). On-line Ramsey numbers for paths and stars. Discrete Math. Theor. Comput. Sci. 10 63-74. MR2445473

[7] Jiang, T., Milans, K. G. and West, D. B. Degree Ramsey number of cycles and blowups of trees, submitted.

[8] Kierstead, H. A. and Konjevod, G. (2009). Coloring number and on-line Ramsey theory for graphs and hypergraphs. Combinatorica 29 49-64. MR2506387

[9] Kinnersley, W. B. Milans, K. G. and West, D. B. (2012). Degree Ramsey numbers of graphs, Combin. Probab. \& Comput. 21 229-253.

[10] Kurek, A. and Rucinski, A. (2005). Two variants of the size Ramsey number. Discuss. Math. Graph Theory 25 141-149. MR2152058

[11] Sauer, N. (1967). Extremaleigenschaften regulärer Graphen gegebener Taillenweite, Österreich. Akad. Wiss. Math.-Natur. Kl. S.-B. II 176 27-43. MR0230646

WILLIAM B. KinNERSLEY

UNIVERSITY OF ILLINOIS

URBANA, IL

USA

E-mail address: wkinners@gmail.com 
Douglas B. West

UNIVERSITY OF ILLINOIS

URBANA, IL

USA

E-mail address: west@math.uiuc.edu

Received OCtober 25, 2011 